BULLETIN OF THE

AMERICAN MATHEMATICAL SOCIETY

Volume 79, Number 3, May 1973

\title{
DEGREE THEORY FOR NONCOMPACT MULTIVALUED VECTOR FIELDS
}

\author{
BY W. V. PETRYSHYN ${ }^{1}$ AND P. M. FITZPATRICK ${ }^{2}$ \\ Communicated by François Treves, October 27, 1972
}

Introduction. In this note we indicate the development and state the properties of a degree theory for a rather general class of multivalued mappings, the so-called ultimately compact vector fields, and then use this degree to obtain fixed point theorems. As will be seen, these results unite and extend the degree theory for single-valued ultimately compact vector fields in [13] and the degree theory for multivalued compact vector fields in ([5], [8]) and also serve to extend to multivalued mappings the fixed point theorems for single-valued mappings obtained in [1], [2], [3], $[9],[10],[13]$, and others (see [13]) and to more general multivalued mappings the fixed point theorems in [4], [6], [8]. The detailed proofs of the results presented in this note will be published elsewhere.

1. Let $X$ be a metrizable locally convex topological vector space. If $D \subset X$ we denote by $K(D)$ and $C K(D)$ the family of closed convex, and the family of compact convex subsets of $D$, respectively. We also use $\bar{D}$ (or cl $D$ ), $\partial D$, and clco $D$ to denote the closure, boundary and convex closure of $D$, respectively. To define what we mean when we say that the upper semicontinuous (u.s.c.) mapping $T: D \rightarrow K(X)$ is ultimately compact, we employ a construction of a certain transfinite sequence $\left\{K_{\alpha}\right\}$ utilized by Sadovsky $[13]$ in his development of the index theory for ultimately compact singlevalued vector fields. Let $K_{0}=$ clco $T(D)$, where $T(A)=\bigcup_{x \in A} T(x)$ for $A \subset D$. Let $\eta$ be an ordinal such that $K_{\beta}$ is defined for $\beta<\eta$. If $\eta$ is of the first kind we let $K_{\eta}=$ clco $T\left(D \cap K_{\eta-1}\right)$, and if $\eta$ is of the second kind we let $K_{\eta}=\bigcap_{\beta<\eta} K_{\beta}$. Then $\left\langle K_{\alpha}\right\rangle$ is well defined and such that $K_{\alpha} \subset K_{\beta}$ if $\alpha>\beta$. Consequently, there exists an ordinal $\gamma$ such that $K_{\beta}=K_{\gamma}$ if $\beta \geqq \gamma$. We define $K=K(T, D)=K_{\gamma}$ and observe that clco $T(K \cap D)=K$. The mapping $T$ is called ultimately compact if either $K \cap D=\varnothing$ or if $T(K \cap D)$ is relatively compact.

Definition 1 . Let $D \subset X$ be open with $T: \bar{D} \rightarrow K(X)$ ultimately compact and such that $x \notin T(x)$ if $x \in \partial D$. If $K(T, \bar{D}) \cap D=\varnothing$ we define $\operatorname{deg}(I-$ $T, D, 0)=0$, and if $K(T, \bar{D}) \cap D \neq \varnothing$ we let $\rho$ be a retraction of $X$ onto $K(T, \bar{D})$ and define

AMS (MOS) subject classifications (1970). Primary 47H10; Secondary 47H99.

Key words and phrases. Multivalued mappings, degree theory, fixed points, measure of noncompactness.

${ }^{1}$ Supported in part by the NSF grant GP-20228.

${ }^{2}$ Supported in part by the NSF grant GP-27719.

Copyright (C) American Mathematical Society 1973 


$$
\operatorname{deg}(I-T, D, 0)=\operatorname{deg}_{c}\left(I-T \rho, \rho^{-1}(D), 0\right),
$$

where the right-hand side of (1) means the topological degree defined in [8] for multivalued compact vector fields.

Note that the right-hand side of (1) is well defined since $x \in D$ and $x \in T(x)$ if and only if $x \in \operatorname{cl}\left(\rho^{-1}(D)\right)$ and $x \in T(\rho(x))$, and one may show that this definition is independent of the particular retraction chosen. The combination of retractions and Leray-Schauder degree has been previously used by F. E. Browder in defining a fixed-point index, and by R. D. Nussbaum in defining the degree for single-valued $k$-set-contractions with $k<1$. We add that if $T$ is compact (i.e., $T: \bar{D} \rightarrow K(X)$ is u.s.c. and $T(\bar{D})$ is relatively compact), then $\operatorname{deg}(I-T, D, 0)=\operatorname{deg}_{c}(I-T, D, 0)$. Furthermore, this degree has the following properties.

THEOREM 1. If $X, D$, and $T$ are as in Definition 1, then the degree given by (1) is such that

(a) if $\operatorname{deg}(I-T, D, 0) \neq 0$, then $T$ has a fixed point in $D$;

(b) if $H: \bar{D} \times[0,1] \rightarrow K(X)$ is u.s.c., $H\left(\bar{D} \cap K^{\prime} \times[0,1]\right)$ is relatively compact where $K^{\prime}=K(H, \bar{D} \times[0,1])$, and $x \notin H_{t}(x)$ for $x \in \partial D$ and $t \in[0,1]$, then $\operatorname{deg}\left(I-H_{0}, D, 0\right)=\operatorname{deg}\left(I-H_{1}, D, 0\right)$;

(c) if $D=D_{1} \cup D_{2}$, where $D_{1}$ and $D_{2}$ are open and $D_{1} \cap D_{2}=\varnothing$, and $x \notin T(x)$ for $x \in \partial D_{1} \cup \partial D_{2}$, then $\operatorname{deg}(I-T, D, 0)=\operatorname{deg}\left(I-T, D_{1}, 0\right)$ $+\operatorname{deg}\left(I-T, D_{2}, 0\right)$

(d) if $D$ is a symmetric neighborhood of the origin and $T: \bar{D} \rightarrow K(X)$ is an odd ultimately compact map with $x \notin T(x)$ for $x \in \partial D$, then $\operatorname{deg}(I-T, D, 0)$ is odd.

We add that even in the case of compact multivalued maps the assertion (d) is new for, unlike [5], [8], we do not require $D$ to be convex.

2. To indicate the usefulness of the topological degree given by Definition 1 we state some examples of ultimately compact maps and some conditions under which the degree is nonzero, a condition which guarantees the existence of fixed points.

If $\left\{p_{\alpha} \mid \alpha \in A\right\}$ is a family of seminorms which determines the topology on $X, \alpha \in A$ and $\Omega \subset X$, then we define $\gamma_{\alpha}(\Omega)=\inf \{d>0 \mid \Omega$ can be covered by a finite number of sets of $p_{\alpha}$-diameter less than $\left.d\right\}$ and $\chi_{\alpha}(\Omega)$ $=\left\{r>0 \mid \Omega\right.$ can be covered by a finite number of $p_{\alpha}$-balls of radius less than $r\}$. Letting $C=\{f: A \rightarrow[0, \infty]\}$, with $C$ ordered pointwise, we define $\gamma: 2^{X} \rightarrow C$ and $\chi: 2^{X} \rightarrow C$ by $\gamma(\Omega)(\alpha)=\gamma_{\alpha}(\Omega)$ and $\chi(\Omega)(\alpha)=\chi_{\alpha}(\Omega)$ for each $\alpha \in A$ and $\Omega \subset X$. Then $\gamma$ and $\chi$ are measures of noncompactness which possess the usual properties (see [14] for $\chi$ ) and we let $\Phi$ denote either $\chi$ or $\gamma$. A u.s.c. map $T: D \rightarrow C K(X)$ is called $\Phi$-condensing if $\Phi(T(A))$ $\Phi(A)$ when $A \subset D$ and $\Phi(A) \neq 0$ and, if $k \in R, T$ is called a $k$ - $\Phi$-con- 
traction if $T(D)$ is bounded and $\Phi(T(A)) \ngtr k \Phi(A)$ when $A \subset D$. Recall that when $X$ is a Banach space, then $T: D \rightarrow C K(X)$ is called contractive (nonexpansive) if there exist $\alpha \in(0,1)(\alpha=1)$ such that

$$
d^{*}(T(x), T(y)) \leqq \alpha d(x, y) \text { for } x, y \in D,
$$

where $d^{*}$ is the Hausdorff metric on $C K(X)$ derived from $d=\|\|$. Finally, following [7], we say that a u.s.c. map $T: D \rightarrow K(X)$ is generalized condensing if for each $Q \subset D$ such that $T(Q) \subset Q$ and $Q \backslash$ clco $T(Q)$ is relatively compact, the set $\bar{Q}$ is compact.

It is clear that every generalized condensing map $T: D \rightarrow D$ is ultimately compact if $D$ is closed and convex. Further, if $X$ is also complete (i.e., a Fréchet space), then every $k-\phi$-contraction $T: \bar{D} \rightarrow C K(X)$ with 0 $<k<1$ is $\phi$-condensing, and every $\phi$-condensing map is ultimately compact.

Theorem 2. Let $D \subset X$ be convex and open and let $T: \bar{D} \rightarrow K(\bar{D})$ be ultimately compact with $K(T, \bar{D}) \neq \varnothing$ and $x \notin T(x)$ for $x \in \partial D$. Then $\operatorname{deg}(I-T, D, 0)=1$, and so $T$ has a fixed point.

It is not hard to show that if $T$ in Theorem 2 is generalized condensing (and, in particular, if $T$ is $\phi$-condensing and $X$ also complete), then $K(T, \bar{D}) \neq \varnothing$ and so Theorem 2 is valid for these classes of maps without the explicit assumption that $K(T, \bar{D}) \neq \varnothing$.

THeOREM 3. Let $X$ be a Fréchet space, $D$ a neighborhood of 0 , and $T: \bar{D} \rightarrow C K(X) a$-condensing map such that

$$
\{\lambda x\} \cap T(x)=\varnothing \quad \text { for } x \in \partial D \text { and } \lambda \geqq 1 .
$$

Then $T$ has a fixed point.

Using Theorem 3 one proves the following general fixed point theorem for $1-\phi$-contractions.

THEOREM 4. Let $X$ and $D$ be as in Theorem 3 and let $T: \bar{D} \rightarrow K(X)$ be a $1-\phi$-contraction. Suppose further that if there is a sequence $\left\{x_{n}\right\} \subset \bar{D}$ with corresponding $y_{n} \in T\left(x_{n}\right)$ for each $n$ such that $x_{n}-y_{n} \rightarrow 0$ as $n \rightarrow \infty$, then there exists $x \in \bar{D}$ with $x \in T(x)$. If T satisfies (2), then Thas a fixed point in $\bar{D}$.

For $X$ a Hausdorff 1.c.t.v.s. and $T$ single-valued, Theorem 2 was proved by Sadovsky [13]. For $X$ a Banach space and $T$ single-valued and $\gamma$ condensing, Theorem 3 was deduced in $[\mathbf{1 0}]$ from the index theory for $\gamma$-condensing maps developed in [9]; for multivalued $\chi$-condensing maps, Theorem 3 includes the result of [6], while for multivalued compact maps with $D=B(0, r)$, Theorem 3 was proved in [4] for $X$ a Banach space and in [8] for $D$ a neighborhood of 0 in a Hausdorff 1.c.t.v.s. In case $X$ is Banach 
and $T$ single-valued, Theorem 4 reduces to Theorem 1 in Petryshyn [11].

Our next result extends to condensing maps $T: \bar{D} \rightarrow K(X)$ and to symmetric but not necessarily convex sets $D$ the validity of the antipodes theorem established in [4], [8] for compact multivalued maps and in [13] for single-valued condensing maps.

THEOREM 5. Let $X$ be a Fréchet space, $D$ a symmetric neighborhood of $0 \in X$, and $T: \bar{D} \rightarrow C K(X) \phi$-condensing. Assume also that

$$
\{x-T(x)\} \cap \lambda\{-x-T(-x)\}=\varnothing \quad \text { for } x \in \partial D \text { and } \lambda \in[0,1] .
$$

Then $\operatorname{deg}(I-T, D, 0)$ is an odd integer.

Since a contraction $T: X \rightarrow C K(X)$ is $\alpha$-ball-contractive, $0<\alpha<1$, on each bounded set in $X$, an immediate consequence of Theorems 3 and 5 is the following corollary.

CoRollary 1. Let $X$ be a Banach space, $D$ a bounded neighborhood of $0 \in X, S: X \rightarrow C K(X)$ contractive, and $C: \bar{D} \rightarrow C K(X)$ compact. If $T$ $=S+C: \bar{D} \rightarrow C K(X)$ satisfies either (2) or (3) on $\partial D$, then $T$ has a fixed point in $T$.

If in Corollary 1 the map $S$ is defined only on $\bar{D}$, then the conclusion still holds provided that either $S$ is single-valued, or the constant $\alpha$ in (1) is $<\frac{1}{2}$, or $X$ is a Hilbert space and $D=B(0, r)$.

We add in passing that if the Banach space is assumed to have the socalled Opial property, $D$ is weakly compact, and $C: \bar{D} \rightarrow C K(X)$ is completely continuous, then Corollary 1 also holds for $S: X \rightarrow C K(X)$ nonexpansive.

We end our note with the following mapping theorem which extends the corresponding result of $\mathrm{Ma}[\mathbf{8}]$ for multivalued compact maps.

THEOREM 6. Let $X$ be a Fréchet space, $D \subset X$ an open set, and $T: D \rightarrow K(X)$ $k-\phi$-contractive with $k<1$. If T is a boundary map in the sense of $M a[8]$, then $(I-T)(D)$ is open.

\section{REFERENCES}

1. F. E. Browder, Nonlinear operators and nonlinear equations of evolution in Banach spaces, Proc. Sympos. Pure Math., vol. 18, part 2, Amer. Math. Soc., Providence, R.1. (to appear).

2. G. Darbo, Punti uniti in transformazioni a condominio non compatto, Rend. Sem. Mat. Univ. Padova 24 (1955), 84-92. MR 16, 1140.

3. M. Furi and A. Vignoli, On $\alpha$-nonexpansive mappings and fixed points, Rend. Accad. Naz. Lincei 48 (1970), 195-198.

4. A. Granas, Theorem on antipodes and theorems on fixed points for a certain class of multi-valued mappings in Banach spaces, Bull. Acad. Polon. Sci. Sér. Sci. Math. Astr. Phys. 7 (1959), 271-275. MR 22 \#8365.

5. - Sur la notion du degré topologique pour une certaine classe de transformations multivalentes dans les espaces de Banach, Bull. Acad. Polon. Sci. Sér. Sci. Math. Astr. Phys. 7 (1959), 191-194. MR 21 \# 7457. 
6. C. J. Himmelberg, J. R. Porter and F. S. Van Vleck, Fixed point theorems for condensing multifunctions, Proc. Amer. Math. Soc. 23 (1969), 635-641. MR 39 \# 7480.

7. E. A. Lifsic and B. N. Sadovsky, A fixed-point theorem for generalized condensing operators, Dokl. Akad. Nauk SSSR 183 (1968), 278-279=Soviet Math. Dokl. 9 (1968), 1370-1372. MR 38 \# 5083.

8. T. W. Ma, Topological degrees of set-valued compact vector fields in locally convex spaces, Dissertiones Math. 92 (1972), 1-43.

9. R. D. Nussbaum, The fixed point index for local condensing maps, Ann. Mat. Pura Appl. 89 (1971), 217-258.

10. W. V. Petryshyn, Structures of the fixed point sets of $k$-set-contractions, Arch. Rational Mech. Anal. 40 (1971), 312-328.

11. - A new fixed point theorem and its applications, Bull. Amer. Math. Soc. 78 (1972), $225-230$.

12. S. Reich, Fixed points in locally convex spaces, Math. Z. 125 (1972), 17-31.

13. B. N. Sadovsky, Measures of noncompactness and condensing operators, Problemy Mat. Anal. Slož. Sistem. 2 (1968), 89-119. (Russian)

14. - Ultimately compact and condensing mappings, Uspehi Mat. Nauk 27 (1972), 81-146. (Russian)

Department of Mathematics, Rutgers University, NeW Brunswick, NeW Jersey 08903

New York University, Courant Institute of Mathematical Sciences, New York, NEW YORK 10012 